the ultrasound examination had aneurysms of maximum diameters of $3 \cdot 3,4 \cdot 7,5 \cdot 2$, and $8 \cdot 0 \mathrm{~cm}$. The patient with the small aneurysm is being reviewed six monthly'; the other three patients have had elective surgical replacement of their aneurysims.

JACK COLLIN JACKIE WALTON

Nuffield Department of Surgery

University of Oxford.

ohn Radcliffe Hospital

Oxford OX3 9DU

1 Collin J, Walton J. Is abdominal aortic aneurvsm familial? Br Med f 1989;299:493. (19 August.)

Tilson MD, Seashore MR. Human genetics of the abdomin aortic aneurysm. Surg Gynecol Obstet 1984;158:129-32.

3 Collin J, Araujo L, Watton J, Lindsell D. Oxford screenin programme for abdominal aortic aneurysm in men aged 65 to 74 years. Lancet 1988 ;ii:613-5.

4 Collin J. The epidemiology of abdominal aortic aneurysm. Br f Hosp Med 1988;40:64-7.

5 Collin J, Araujo L, Walton J. How fast do very small abdominal aortic aneurysms grow? European fournal of l'ascular Surgery $1989: 3: 15-7$

\section{Increased thermogenesis in children with type I diabetes}

SIR,-Dr M J Müller and colleagues showed increased thermogenesis during adrenaline infusion and consequent raised urinary nitrogen excretion in diabetic children.' This finding confirms our previous observation. ${ }^{2}$ We measured the thermic effect of food for three hours in six newly diagnosed and six chronically treated diabetic children using indirect calorimetry. The children were receiving continuous subcutaneous insulin infusion. Increased thermogenesis was observed in both groups but especially in the newly diagnosed children compared with nine controls 5.02 (SE 0.89) $\mathrm{kJ} / \mathrm{kg}$ lean body mass $/ 3 \mathrm{~h} v 2.86(0.35) \mathrm{p}<0.05$. The relative contribution of protein to resting energy expenditure was significantly higher in the diabetic children (newly diagnosed 27.9 (4.5)\%; chronically treated $23.4(2.6 \%)$ than in the controls $(13.9(1.6 \%) ; \mathrm{p}<0.05)$.

Dr Müller and his coworkers also stated that the degree of glycaemic control did not influence the thermic effect of adrenaline. This part of their observation, however, is not convincing as the two groups compared had similar high blood glucose concentrations both before and during adrenaline infusion. Furthermore, their statement contradicts our earlier results concerning the effect of rapid metabolic decompensation on thermogenesis induced by diet. ${ }^{3}$ Resting energy expenditure and thermogenesis induced by diet were measured in 12 diabetic children while they received continuous subcutaneous insulin infusion and again on a separate day two hours after stopping continuous subcutaneous insulin infusion. The short term insulin insufficiency resulted in hyperglycaemia $(5 \cdot 1(0.5) \mathrm{mmol} / \mathrm{l}$ on day $1 v 16 \cdot 2(1 \cdot 6)$ $\mathrm{mmol} / \mathrm{l}$ on day $2 ; \mathrm{p}<0.01)$ and was associated with rising resting energy expenditure $(5.6(0.3) v 6.4$ $(0.4) \mathrm{kJ} / \mathrm{kg}$ lean body mass $/ \mathrm{h} ; \mathrm{p}<0.05)$ and with a significantly reduced thermogenesis induced by $\operatorname{diet}(4 \cdot 7(0.6) v 3.3(0.4) \mathrm{kJ} / \mathrm{kg}$ lean body mass $/ 3 \mathrm{~h}$; $\mathrm{p}<0.01)$. We conclude that even tight glycaemic control with continuous subcutaneous insulin infusion treatment does not mean optimal metabolic control, and therefore further refinement of the treatment of diabetes mellitus is necessary.

D MOLNÁR

T DECSI

Department of Paediatrics,

G SOLTÉSZ

7623 Pécs, Hungary

1 Müller MJ, Mühler A, Lautz HU, Schmidt FW, Daiber M, Hürter $P$. Energy expenditure in children with type I diabetes: evidence for increased thermogenesis. Br Med f 1989;299: 487-91. (19 August.)

2 Molnár D, Decsi T, Soltész G. Resting energy expenditure and food-induced thermogenesis in diabetic children receiving continuous subcutaneous insulin infusion. Diabetes Res 1988; 7:117-21

3 Decsi T, Molnár D, Soltész G, Mestyán J. Abnormal thermogenesis in diabetic children receiving continuous subcutaneous insulin infusion therapy [Abstract]. Ped Res 1986;20:1046.

\section{Antenatal screening for syphilis}

SIR, - We agree with many of the arguments of Dr Jennifer C Clay as to why screening should continue, ' and wish to contribute to this debate by considering the issue from an obstetric rather than a genitourinary or even paediatric viewpoint.

Firstly, we cannot have pride in our achievements as long as prevention by the present screening methods remains ineffective. This failure is shown by the data of Holland and O'Mahony ${ }^{2}$ described in their letter. ${ }^{3}$

Secondly, Dr Clay lists several reasons for failure of first trimester screening, including failure to retest women at high risk. High risk is a nebulous concept, and establishing adequate criteria is even more difficult in antenatal clinics than in genitourinary clinics. All women attending genitourinary clinics for whatever reason may be considered high risk in contrast to those attending antenatal clinics. Furthermore, any woman suspected of having a sexually transmitted disease during pregnancy should obviously be retested. Beyond prostitution and drug abuse, however, all other known risk factors have low positive predictive values. Those women with positive serology were predominantly young, single, and unemployed. ${ }^{2}$ This group constitutes a large percentage of women attending antenatal clinics, especially in inner city areas.

Thirdly, obstetricians have the same problem as other doctors in identifying high risk factors and HIV testing. Universal antenatal screening for $\mathrm{HIV}$ is not recommended at present, but careful selective testing (or "case finding") is recommended according to the risk groups as defined from time to time by the Department of Health. ${ }^{+}$We suggest that all women who have been tested for HIV during pregnancy should be retested for syphilis each time they are retested for HIV.

Finally, we recognise that there are potential problems regarding informed consent for selective retesting for syphilis that are similar to those for HIV testing.

Women's Hospital,

JOHN H MACDONALD

Liverpool L8 7N

Mill Road Maternity Hospital

SANJEEV SHARMA

Liverpool L6 2AH

1 Clay JC. Antenatal screening for syphilis. Br Med $\mathcal{f}$ 1989;299: 409-10. (12 August.)

2 Holland EFN, O'Mahony CP. Is it time to review antenatal screening for syphilis? Br $\mathcal{F}$ Obstet Gynaecol 1989;95:1005-6.

3 O'Mahoney $\mathrm{C}$, Holland $\mathrm{N}$. Antenatal screening for syphilis. Br Med f 1989;299:859. (30 September.)

4 Royal College of Obstetricians and Gynaecologists. Report of the RCOG sub-committee on problems associated with AIDS in relation to obstetrics and prnaecology. 3. HIV and pregnancy. London: RCOG, 1987:5-6.

SIR, - Dr Jennifer C Clay's editorial' prompted us to review our experience of the contribution of antenatal screening to the diagnosis of syphilis. Between January 1986 and August 1989, 11 heterosexual men, five homosexual men, and six women with early syphilis were treated in this department. Three of the six women were referred after positive serological tests for syphilis in an antenatal clinic, two attended as a result of contact tracing, and one was diagnosed by routine testing in this department. None of the three women referred from an antenatal clinic had had symptoms suggesting early syphilis.

As a result of contact tracing four sexual partners of the three women presented to us. Syphilis was excluded in three of them, but secondary syphilis was diagnosed in one. This man had had a nonpruritic skin rash treated with antibiotics three months previously and was awaiting biopsy of a persistent oral (snail tract) ulcer. Syphilis had not been considered in the differential diagnosis of the rash or the ulcer.

Because the symptoms of early syphilis may not be noticed by patients and the signs may be overlooked by doctors, some cases of early syphilis will be diagnosed only by serological tests for syphilis. In the past three and a half years nearly a quarter of cases of heterosexually acquired early syphilis have presented to us as a result of antenatal screening, and we support Dr Clay's opinion that this should be continued.

K SHANMUGARATNAM P G WATSON

Department of Genitourinary Medicine,

Newcastle General Hospital.

Newcastle upon Tyne NE46BE

1 Clay JC. Antenatal screening for syphilis. Br Med f 1989;299: 409-10. (12 August.)

\section{Antenatal prophylaxis with anti-D immunoglobulin}

SIR,-Dr Ruth Hussey ${ }^{1}$ does not contest our findings ${ }^{2}$ that antenatal $\mathrm{Rh}$ prophylaxis reduces the incidence of $\mathrm{Rh}$ sensitisation but has reservations about its general application.

Firstly, she contends that as anti-D immunoglobulin is already recommended for possible bleeding episodes such as amniocentesis its routine use antenatally is unnecessary. Unfortunately, most new cases of sensitisation show antibodies for the first time between 30 and 40 weeks' gestation without any history of such sensitising episodes, and therefore anti-D immunoglobulin would not have been given.

Secondly, Dr Hussey claims that routine antenatal prophylaxis is unnecessary for a third of cases as the baby will be $\mathrm{Rh}$ negative and that the presence of passive anti-D immunoglobulin in the mother's serum towards the end of pregnancy can cause confusion and additional expensive antenatal monitoring; we did not find this a problem in the Yorkshire trial. Her main objection is the question of safety. Obviously, we cannot state categorically that in 20 years' time there may not be sequelae, but we note that in Canada antenatal prophylaxis has been practised for more than 10 years without any detectable side effects. ${ }^{3}$

Finally, Dr Hussey claims that we gave little detail about the severity of the rhesus disease in the affected babies of mothers immunised during pregnancy. We had dealt with this in the original trial report, ${ }^{4}$. where among the control group there were 28 mildly affected infants requiring only phototherapy and five moderately or severely affected ones who required exchange transfusion. We could not accurately update these figures in the present paper because we examined only obstetric records. Nevertheless, among the control pregnancies reported for the first time there were two more infants who had required exchange transfusions. Among the babies of immunised mothers in the trial group there were none requiring exchange transfusion, although there was one unexplained stillbirth already reported ${ }^{4}$ that was probably unrelated to rhesus disease. Of relevance is a recent study by Clarke and Mollison showing that 25-30 babies a year are still lost because of rhesus disease.

We have always claimed that routine antenatal prophylaxis is necessary only for the first pregnancy, or if the first infant was rhesus negative possibly for the second pregnancy, so a threefold increase in usage is unlikely. If high risk patients could be identified then routine administration would be unnecessary, but there are no means of 